\title{
The Cosmic Background Imager
}

\author{
T. J. Pearson, B. S. Mason, S. Padin, A. C. S. Readhead, \\ M. C. Shepherd, J. Sievers, P. S. Udomprasert and J. K. Cartwright \\ California Institute of Technology, Pasadena, CA 91125, USA
}

\begin{abstract}
The Cosmic Background Imager (CBI) is an instrument designed to make images of the cosmic microwave background radiation and to measure its statistical properties on angular scales from about 3 arc minutes to one degree (spherical harmonic scales from $l \sim 4250$ down to $l \sim 400$ ). The CBI is a $13-$ element interferometer mounted on a 6 meter platform operating in ten $1-\mathrm{GHz}$ frequency bands from $26 \mathrm{GHz}$ to $36 \mathrm{GHz}$. The instantaneous field of view of the instrument is 45 arcmin (FWHM) and its resolution ranges from 3 to 10 arcmin; larger fields can be imaged by mosaicing. At this frequency and resolution, the primary foreground is due to discrete extragalactic sources, which are monitored at the Owens Valley Radio Observatory and subtracted from the CBI visibility measurements.

The instrument has been making observations since late 1999 of both primordial CMB fluctuations and the Sunyaev-Zeldovich effect in clusters of galaxies from its site at an altitude of 5080 meters near San Pedro de Atacama, in northern Chile. Observations will continue until August 2001 or later. We present preliminary results from the first few months of observations.
\end{abstract}

\section{Introduction}

The Cosmic Background Imager (CBI) is a radio interferometer with thirteen 0.9-meter diameter antennas mounted on a 6 -meter tracking platform. It operates in ten 1-GHz frequency channels from 26 to $36 \mathrm{GHz}$. Like several other instruments described at this Symposium, it is dedicated primarily to the measurement of the angular power spectrum $C_{l}$ of the cosmic microwave background radiation (CMBR). The CBI is sensitive to multipoles in the range $400<l<4250$. The low end of this range is also covered by the recent results from Boomerang (de Bernardis et al. 2000; Lange et al. 2000) and Maxima (Hanany et al. 2000; Balbi et al. 2000), so the CBI will be able to check those results and extend the spectrum to higher $l$. The Degree Angular Scale Interferometer (DASI), described by Erik Leitch at this Symposium, is a lower-resolution sister project to the CBI, covering $140<l<900$. The DASI and the CBI share many design elements, and the telescope control software and the CBI correlator and receiver control electronics have been duplicated by the DASI team for use with the DASI project.

In addition to measuring the CMBR power spectrum, the CBI is being used to image the Sunyaev-Zeldovich effect in clusters of galaxies, to detect or place limits on the polarization of the CMBR, and to characterize foreground emission in the $26-36 \mathrm{GHz}$ band. We have also used it to image supernova remnants, $\mathrm{H}$ II regions, and other discrete sources. 
As the techniques involved in interferometric observations of the CMBR are very different from those used in total-power and switched-beam experiments, interferometers are a valuable alternative to such experiments. A single interferometer baseline is sensitive to a narrow range of spatial frequencies, and so the square of the measured visibility provides a direct estimate of the power spectrum in a corresponding range of $l$. The center of this range is $l \approx 2 \pi u$, where $u$ is the length of the interferometer baseline in wavelengths, and the width of the range is set by the size of the antenna aperture, with smaller antennas giving higher resolution in $l$. The instantaneous field of view ("primary beam") of the interferometer is inversely proportional to the size of the antenna. Thus by adjusting the baseline lengths and the size of the apertures it is possible to match the characteristics of the instrument to the scientific goals. Two major advantages of interferometers are that they are insensitive to extended emission, including the isotropic background and much of the atmospheric emission, and that most systematics (receiver gain instabilities, for example) are uncorrelated between antennas and thus do not affect the measurements significantly. Interferometers make images of the sky emission convolved with an accurately known, adjustable point-spread function.

\section{Instrument Design}

The CBI is shown in Figure 1, and its major characteristics are shown in Table 1. More details of the design may be found in three papers by Padin et al. (2000a, b, d), and on the CBI web page (http://www.astro.caltech.edu/ tjp/CBI/).

The CBI operates in the frequency band $26-36 \mathrm{GHz}$, which was chosen as a compromise between the effects of astronomical foregrounds and atmospheric emission and the sensitivity that can be achieved with HEMT amplifiers.

The key design challenges in the project were eliminating cross-talk in a compact array and developing a wide-band correlator. Receiver noise scattering between adjacent antennas (cross-talk) causes false signals at the correlator output and this could limit the sensitivity of the instrument. We developed a shielded Cassegrain antenna with low scattering to reduce the cross-talk (Padin et al. 2000a). The antennas have machined, cast aluminum primaries which sit at the bottom of deep cylindrical shields. The upper rims of the shields are rolled with a radius of a few wavelengths to reduce scattering from the shield itself. The secondaries are made of carbon fiber epoxy, to minimize weight, and supported on transparent polystyrene feed legs. Cross-talk between the antennas is $<-110 \mathrm{~dB}$ in any CBI band.

The antennas are mounted on a rigid tracking platform supported by an altazimuth mount that is fully steerable to elevations $>42^{\circ}$. The antenna platform can be rotated about the optical axis. In normal observations, the platform tracks the parallactic angle so that observations are made at fixed $(u, v)$ points: i.e., the baseline orientations are fixed relative to the sky. Additional discrete steps in the orientation of the platform are used to change the baseline orientations and thus sample more $(u, v)$ points.

All the electronics, including the local oscillator systems and the correlators, are also mounted on the antenna platform. Because the antennas do not move relative to one another, all the receivers have equal, stable paths to the correlator 


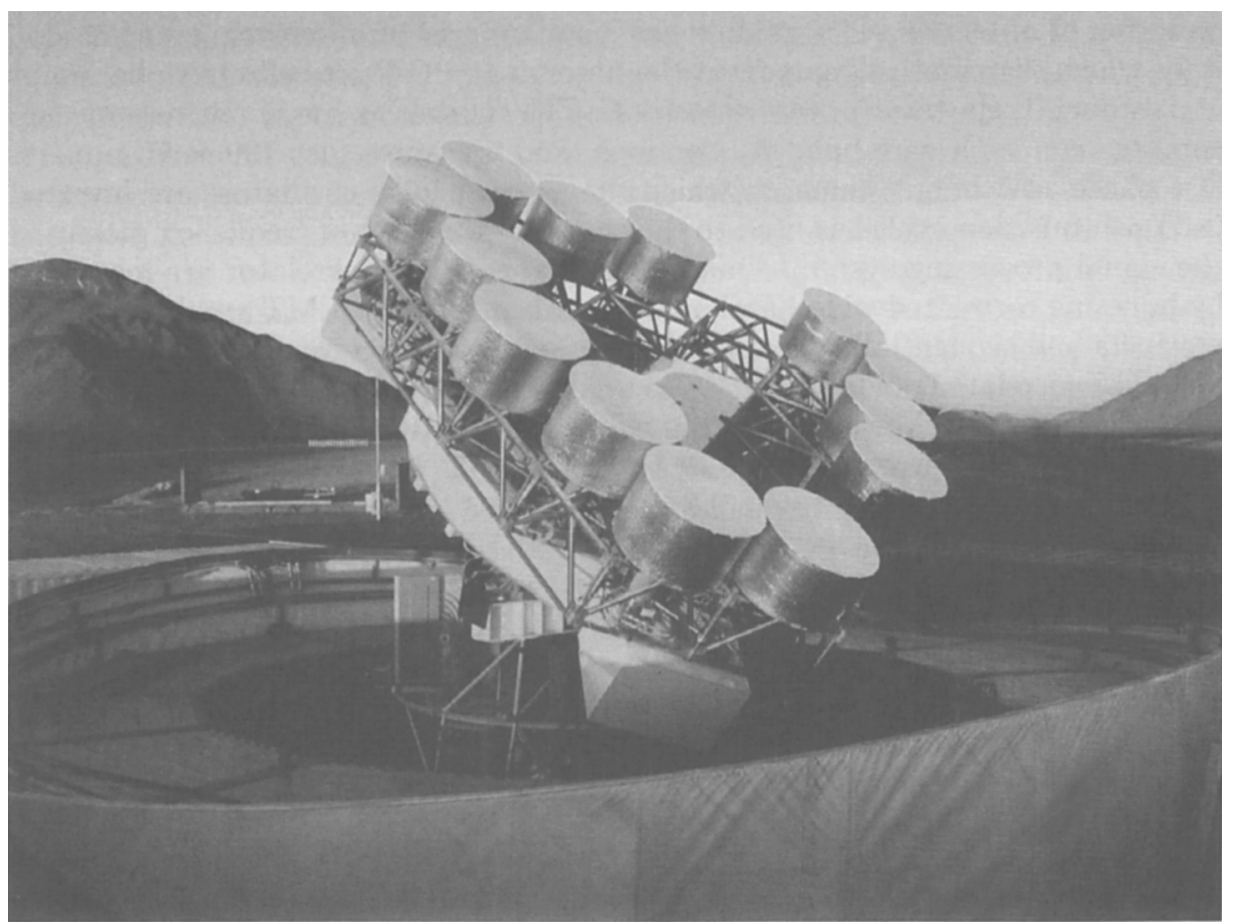

Figure 1. View of the CBI on the Llano de Chajnantor, with the antennas in a ring configuration.

and no delay tracking is required. The correlator (Padin et al. 2000b) is an analog filter bank correlator with ten $1-\mathrm{GHz}$ bands. The design is based on a hybrid module that contains all the signal distribution and multipliers for a $1-\mathrm{GHz}$

Table 1. CBI Specifications

\begin{tabular}{ll}
\hline Observing frequency: & $26-36 \mathrm{GHz}$ (wavelength $\sim 1 \mathrm{~cm})$ \\
Number of channels: & 10 channels, each $1 \mathrm{GHz}$ wide \\
Number of antennas: & 13 \\
Number of baselines: & 78 \\
Receivers: & HEMT amplifiers, cooled to $6 \mathrm{~K}$ \\
Correlator: & 780 analog complex correlators \\
Antenna: & Cassegrain, $0.90 \mathrm{~m}$ diameter \\
Primary beam: & Gaussian FWHM 45 arcmin \\
Minimum baseline: & $1.00 \mathrm{~m}(l \sim 630)$ \\
Maximum baseline: & $5.51 \mathrm{~m}(l \sim 3500)$ \\
Synthesized beamwidth: & FWHM $3-10$ arcmin \\
System temperature: & $25 \mathrm{~K}$ \\
Noise in visibility measurements: & $4.5 \mathrm{Jy} \mathrm{s} / 2 \mathrm{rms}$ in each 1-GHz channel \\
Expected signal $\left(T_{\mathrm{rms}}=40 \mu \mathrm{K}\right):$ & $\sim 44 \mathrm{mJy} \mathrm{rms} \mathrm{on} \mathrm{1-m} \mathrm{baseline}$ \\
\hline
\end{tabular}


band for 13 antennas. The module has a square grid of microstrip transmission lines which distribute signals from the antennas to Gilbert cell multiplier chips and tunnel-diode total power detectors. The module is made entirely of chip components, with wire-bond connections, and measures just $100 \times 70 \mathrm{~mm}$. A fast phase-switching scheme, in which the receiver local oscillators are inverted in Walsh function cycles, is used to reject cross-talk and low-frequency pickup in the signal processing system. Quadrature errors in the correlator are measured by injecting correlated wide-band noise just ahead of the HEMT amplifiers in the receivers and sequentially changing the phase of each receiver local oscillator by $90^{\circ}$. The correlated noise source is also used to measure gain and phase variations in the instrument. Variations in the calibration of the CBI, after applying the noise calibration corrections, are at the $1 \%$ and $1^{\circ}$ level. The efficiency of the receiving system is in the range $0.8-1$.

The antennas have low-noise broadband HEMT amplifier receivers with $\sim 25 \mathrm{~K}$ noise temperatures. The typical system noise temperature averaged over all ten bands is $\sim 30 \mathrm{~K}$, including ground spillover and atmosphere.

The antennas can be moved from one location to another on the platform to change the baseline lengths and orientations. The results presented here were made in two different configurations; one (shown in Figure 1) with most of the antennas around the perimeter of the platform, which provides a fairly uniform $(u, v)$ coverage and allows easy access to the receivers; and a second, more compact configuration which provides greater sensitivity at low $l$.

One of the 13 receivers is currently configured for right circular polarization while the other 12 receive left circular polarization. The images presented here are from the parallel-polarization baselines, which are sensitive to Stokes' parameter $I$ (assuming circularly-polarized emission is negligible). The 12 crosspolarized baselines are sensitive to Stokes' parameters $Q \pm i U$ and are being used to place limits on the polarization of the CMBR. The ability to rotate the array about the optical axis facilitates polarization calibration and enables us to measure both $Q$ and $U$ at the same $(u, v)$ point.

\section{The Site}

The CBI must be on a high, dry site to ensure that atmospheric brightness fluctuations do not limit the sensitivity of the instrument, and, in addition, the horizon must be low to minimize pickup of ground radiation. We have chosen a site at an altitude of $5080 \mathrm{~m}$ about $40 \mathrm{~km}$ east of San Pedro de Atacama in northern Chile. The site is near the center of the $10-\mathrm{km}$ plateau of Llano de Chajnantor, close to the site proposed for the Atacama Large Millimeter Array (ALMA).

The CBI is housed in a retractable dome to provide some protection from rain, snow and wind. On most days, the wind at Chajnantor peaks late in the afternoon at about $15 \mathrm{~m} / \mathrm{s}(30 \mathrm{mph})$, but we have experienced winds greater than $45 \mathrm{~m} / \mathrm{s}$. Under these conditions wind chill is a severe problem, but the dome provides a sheltered work space where we can repair and maintain the CBI. The dome is a hemispherical steel frame covered with polyester cloth, sitting atop a 2-m high wall $10.5 \mathrm{~m}$ in diameter. 
The site facilities include a control room, laboratory, machine shop, power plant, two bedrooms, and a bathroom - all constructed within standard shipping containers. All the equipment required for repairs and modifications is at the site. Additional containers used for storage are placed around the dome to provide a wind break. To counteract the effects of high altitude, the air in the work and living areas is oxygen-enriched (using molecular sieves to separate oxygen from the air), and people working outside can wear portable oxygen tanks with demand regulators when necessary to improve efficiency and safety. The power plant and fuel tanks are located about $100 \mathrm{~m}$ east of the CBI; the average power consumption for the site is about $100 \mathrm{~kW}$ and the power plant has a pair of diesel generators rated for $150 \mathrm{~kW}$ at $5000 \mathrm{~m}$.

The base facilities are located in the historic oasis town of San Pedro de Atacama at an elevation of $2500 \mathrm{~m}$, and include bedrooms, kitchen, conference room, and computer room; the facilities are provided by the Hosteria La Casa de Don Tomás.

\section{Project Status}

The CBI was designed in 1995-1996, and constructed in 1997-1998. After six months of test observations on the Caltech campus in Pasadena, using six receivers and four 1-GHz correlators, the instrument was partially disassembled, packed and shipped to Chile where it arrived at the Llano de Chajnantor on August 28, 1999. We obtained first light in Chile on November 1, 1999, and the full array of 13 receivers and ten correlators was fully assembled and started routine observations in January 2000. Unfortunately the weather in the first few months of 2000 has been unusually bad, and we have lost about $50 \%$ of the nights owing to cloud, high winds, and snow. In the remaining nights the observing conditions were superb and fluctuations in the atmosphere did not significantly increase the system noise. A minor eruption of the nearby volcano Lascar on July 18 did not interrupt observing.

The preliminary results presented here are from observations made between January and July 2000. We will continue to make observations in Chile until August 2001 or later.

\section{Observing Strategy}

For all likely CMBR power spectra, the expected signal decreases strongly with interferometer baseline length (the expected root-mean-square visibility is proportional to $C_{l}$ ), so we are making observations in two modes: (1) deep observations of selected $45^{\prime}$ fields, concentrating on the higher end of our $l$ range these observations will be limited by instrumental noise and foreground emission; (2) shallower observations of many overlapping fields ("mosaic" observations) to measure the power spectrum at lower $l$ - in these observations we will image enough sky to balance the effects of thermal noise and sample (cosmic) variance. Our observations have been made in the declination range $-5^{\circ}<\delta<-2^{\circ}$ in regions of low IRAS $100 \mu \mathrm{m}$ emission at several galactic latitudes. 
Daytime observations of the CMBR are not possible because radio emission from the sun in the far sidelobes of the antennas contaminates the visibilities; we also restrict observations to fields more than $60^{\circ}$ from the moon.

The dominant systematic contamination in the CBI observations is due to radiation from the ground ("ground spillover"). This introduces spurious signals up to a few Jy on the shortest (1-meter) baselines, but the effect is much less on longer baselines. Fortunately the ground signal is stable and repeatable on short time scales. We remove it by differencing the visibilities measured on two fields observed at the same elevation and azimuth. Usually we alternate between two fields; we observe one "leading" field for $8 \mathrm{~min}$, and then observe a second "trailing" field $8 \mathrm{~min}$ later in right ascension for the next $8 \mathrm{~min}$, so that both are observed at the same position relative to the ground. By Fouriertransforming the visibility differences, we obtain an image of the difference of two fields separated by $8 \mathrm{~min}$ in right ascension. By comparing observations made at different elevations and under different weather conditions, we have found that any residual ground contamination in the difference images is less than a few per cent of the CMBR signal at spatial frequencies corresponding to the 1-meter baselines. While differencing requires longer observing times than undifferenced observations, and can also make it difficult to distinguish a positive $\delta T$ in one field from a negative $\delta T$ in the other field, it does not otherwise impede the measurement of the CMBR power spectrum.

The flux-density scale is based on observations of Jupiter, which has been measured to have an effective temperature of $152 \pm 5 \mathrm{~K}$ at $32 \mathrm{GHz}$ (Mason et al. 1999); as Jupiter does not have a simple thermal spectrum between 26 and $36 \mathrm{GHz}$, we transfer this calibration to other frequencies using observations of Tau A (the Crab nebula) which has a power-law spectrum in this range. Receiver gain and system-temperature variations between observations of the calibration sources are removed by reference to the internal calibration signal which is injected into the front end of each receiver.

We make images from the visibility data by the usual synthesis-imaging procedures (e.g., Taylor, Carilli \& Perley 1999). The sensitivity of an image made from a single pointing is tapered by the primary beam of the antennas, approximately a gaussian of FWHM $45^{\prime} \times(30 \mathrm{GHz} / \nu)$. In the mosaic observations, we typically observe at a grid of positions spaced by $20^{\prime}$ (e.g., 36 positions to map a $2^{\circ} \times 2^{\circ}$ field), and make a weighted linear combination of the individual images (Cornwell 1988). The resulting mosaic image has almost uniform sensitivity except at the edges.

The major foreground contaminant is discrete, unresolved extragalactic sources (galaxies and quasars). In order to remove these sources from the data, we have made $26-34 \mathrm{GHz}$ observations of all the sources in our fields that are stronger than $6 \mathrm{mJy}$ in the $1.4 \mathrm{GHz}$ NRAO VLA Sky Survey (Condon et al. 1998), using the Owens Valley Radio Observatory's 40-meter telescope. We monitor those that are stronger than $6 \mathrm{mJy}$ at $30 \mathrm{GHz}$ using the 40 -meter telescope to obtain their flux densities at the time of the CBI observations. Then the expected responses to these sources are subtracted from the CBI visibility data before imaging.

Figure 2 shows one of our mosaic fields and the effectiveness of our source subtraction technique. The images are dominated by CMBR, and in future work 

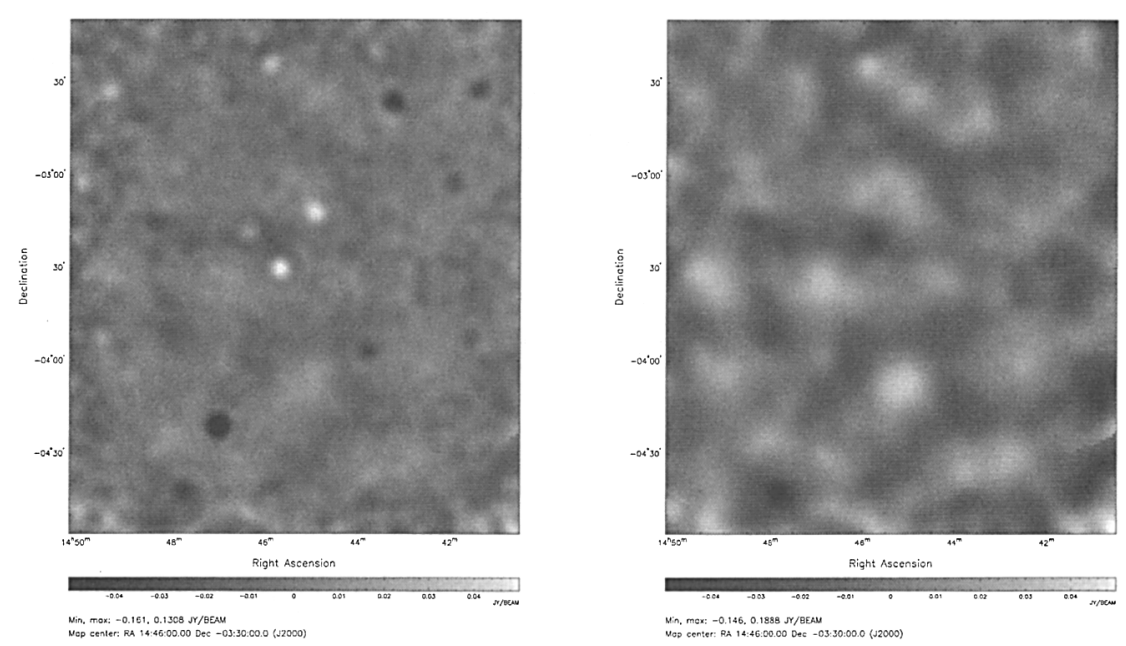

Figure 2. Preliminary mosaic images of a $\sim 2^{\circ}$ field centered at about RA $14^{\mathrm{h}} 46^{\mathrm{m}}$, Decl. $-3^{\circ} 30^{\prime}$. The images show the difference of the emission in this field and one centered at RA $14^{\mathrm{h}} 54^{\mathrm{m}}$. Each field was observed in 50 single pointings, with an integration time of about $2^{\mathrm{h}}$ on each field. Left: Before source subtraction; synthesized beam FWHM 5.2 arcmin. Light spots are discrete sources in the leading field, while dark (negative) spots are discrete sources in the trailing field. Right: After subtracting known sources using flux densities measured with the OVRO 40-meter telescope; resolution reduced to FWHM 7.3 arcmin to improve sensitivity to large-scale emission. In this preliminary image, not all the known sources have yet been subtracted.

we will quantify this. There are a number of potential problems that we need to understand before we are ready to determine the CMBR power spectrum, however. First, we must look for possible systematic errors due to instrumental problems, atmosphere, or residual ground spillover. These systematics are all largely removed by our differencing strategy, but we can look for residual effects by comparing observations made at different times, and on distinct but equal baselines. Such tests have already shown that any contamination is less than a few percent of the CMBR signal. Secondly, it is clear that we can accurately remove the strongest discrete sources, but unsubtracted sources and errors in the flux densities of the subtracted sources will bias the power spectrum. We will measure the number counts of fainter sources and make a statistical correction for them. Thirdly, we have yet to determine how much of the emission seen in our images could be foreground from the Galaxy. The ten CBI frequency bands allow us to constrain the spectrum of the emission, and preliminary results show that the spectrum is consistent with thermal emission. Synchrotron or free-free foreground emission must be much less than the CMBR. 


\section{Estimating the Power Spectrum}

We will use a maximum likelihood method to estimate the power spectrum. If the CMBR fluctuations are gaussian, then the required information about their power spectrum is contained in the covariance matrix $M_{j k}$ of the visibility measurements. The covariance between a visibility measurement $j$ at $(u, v)$ position $\mathbf{u}_{j}$ and frequency $\nu_{j}$ and another measurement $k$ is related to the power spectrum by

$$
M_{j k}=\left\langle V\left(\mathbf{u}_{j}, \nu_{j}\right) V^{*}\left(\mathbf{u}_{k}, \nu_{k}\right)\right\rangle=K_{j} K_{k} \int_{0}^{\infty} W_{j k}(v) C(v) v d v,
$$

where $K_{j} \equiv 2 \nu_{j}^{2} k_{\mathrm{B}} T_{0} g\left(\nu_{j}\right) / c^{2}$ is the conversion factor from $\delta T / T$ to intensity, $C(v)=C_{l}$ for $l \approx 2 \pi v$, and $W_{j k}(v)$ is the window function defined by

$$
W_{j k}(v)=\int_{0}^{2 \pi} \tilde{A}\left(\mathbf{u}_{j}-\mathbf{v}, \nu_{j}\right) \tilde{A}^{*}\left(\mathbf{u}_{k}-\mathbf{v}, \nu_{k}\right) d \theta_{v},
$$

where $\tilde{A}$ is the normalized Fourier transform of the primary beam response (Hobson, Lasenby, \& Jones 1995; White et al. 1999). The diagonal window function, $W_{j j}(v)$, is approximately a gaussian of FWHM 70 wavelengths $(\Delta l \approx 430)$ for the CBI. Using this expression we can compute the expected rms visibility for a flat power spectrum

$$
l(l+1) C_{l} / 2 \pi=\left(\delta T / T_{\mathrm{cmb}}\right)^{2}=\text { constant } .
$$

The result is

$$
\sqrt{\left\langle V^{2}\right\rangle} \approx 1.1 \mathrm{mJy}(d / \mathrm{m})^{-1}(\delta T / \mu \mathrm{K}),
$$

where $d$ is the baseline length. Typical cosmologies have $\delta T=60 \mu \mathrm{K}$ for $l \sim 600$ and $\delta T=15 \mu \mathrm{K}$ for $l \sim 2500$; the corresponding rms visibilities are about 50 mJy and $3 \mathrm{mJy}$.

Additional terms must be included in the covariance matrix to account for instrumental noise (which to a good approximation is uncorrelated between different measurements and can be accurately measured) and other errors including unsubtracted point sources and errors in foreground subtraction. Given the covariance matrix, we can calculate the likelihood of the observed visibility data for a particular model power spectrum.

Observations of mosaiced fields will be used to estimate the power spectrum with increased resolution in $l$. In a mosaic, the $l$ resolution is not limited by the inverse of the width of the primary beam but improves in proportion to the size of the field surveyed. For a field of size $2^{\circ}$, the resolution in $l$ is about 160 , and will enable us to resolve the acoustic peaks. Mosaicing also reduces the cosmic variance as a larger area is being surveyed. The covariance matrix for a mosaic observation is more complicated than that given above, as it involves correlations between different pointing centers as well as those between different baselines and frequencies, and the dimension of the matrix is correspondingly increased, to $>10^{4} \times 10^{4}$ (number of pointings times number of distinct baselines times number of frequency channels). 


\section{Conclusions}

The preliminary results presented here show that the CBI is working well. We will soon submit a paper to the Astrophysical Journal presenting the first quantitative results of our observations on the intrinsic anisotropy of the CMBR at $l<1500$ (Padin et al. 2000c). In subsequent papers we will extend the results to higher $l$, which will require careful attention to the effects of discrete sources, and use mosaic observations to improve the resolution in $l$. While so far we have concentrated on "blank" regions for measurement of the intrinsic power spectrum, we are also observing a sample of galaxy clusters to study the Sunyaev-Zeldovich effect (Udomprasert et al. 2000).

Acknowledgments. We are grateful for the contributions to this project of our our collaborators: Russ Keeney, Steve Miller, Walter Schaal, and John Yamasaki (Caltech); John Carlstrom and Erik Leitch (University of Chicago); Bill Holzapfel (University of California, Berkeley); Steven Myers (National Radio Astronomy Observatory); Marshall Joy (NASA's Marshall Space Flight Center); Angel Otarola (European Southern Observatory); and Leonardo Bronfman, Jorge May, Simon Casassus, and Pablo Altamirano (University of Chile). The CBI project has been supported by the National Science Foundation under grants AST-9413935 and AST-9802989, and we are also grateful for the generous support of Maxine and Ronald Linde, Cecil and Sally Drinkward, and our colleagues at the California Institute of Technology, especially the Provost, the President, and the Chairman of the Division of Physics, Mathematics, and Astronomy. We are grateful to CONICYT for permission to operate the CBI in the Chajnantor Scientific Preserve in Chile. JS and PSU acknowledge support from National Science Foundation Graduate Student Fellowships.

\section{References}

Balbi, A. et al. 2000, ApJ, 545, L1

Condon, J. J., Cotton, W. D., Greisen, E. W., Yin, Q. F., Perley, R. A., Taylor, G. B. \& Broderick, J. J. 1998, AJ, 115, 1693

Cornwell, T. J. 1988, A\&A, 202, 316

De Bernardis, P. et al. 2000, Nature, 404, 955

Hanany, S. et al. 2000, ApJ, 545, L5

Hobson, M. P., Lasenby, A. N. \& Jones, M. E. 1995, MNRAS, 275, 863

Lange, A. E. et al. 2000, Phys.Rev.D, in press (astro-ph/0005004)

Mason, B. S., Leitch, E. M., Myers, S. T., Cartwright, J. K. \& Readhead, A. C. S. 1999, AJ, 118,290

Padin, S., Cartwright, J. K., Joy, M. \& Meitzler, J. C. 2000a, IEEE Trans. Antennas Propagat. 48, 836

Padin, S., Cartwright, J. K., Shepherd, M. C., Yamasaki, J. K. \& Holzapfel, W. L. 2000b, IEEE Trans. Instrum. Meas., submitted

Padin, S., Cartwright, J. K., Mason, B. S., Pearson, T. J., Readhead, A. C. S., Shepherd, M. C., Sievers, J., Udomprasert, P. S., Holzapfel, W. L., Myers, S. T., Carlstrom, J. E., Leitch, E. M., Joy, M., Bronfman, L. \& May, J. 2000c, ApJ, submitted

Padin, S. et al. 2000d, in preparation 
Taylor, G. B., Carilli, C. L. \& Perley, R. A. (ed.) 1999, Synthesis Imaging in Radio Astronomy II, ASP Conf. Ser. Vol. 180 (San Francisco: ASP)

White, M., Carlstrom, J. E., Dragovan, M. \& Holzapfel, W. L. 1999, ApJ, 514, 12

Udomprasert, P. S., Mason, B. S. \& Readhead, A. C. S. 2000, in Constructing the Universe with Clusters of Galaxies, ed. F. Duret \& D. Gerbel, in press 